\title{
Human pericytes adopt myofibroblast properties in the microenvironment of the IPF lung
}

\author{
Parid Sava, ${ }^{1}$ Anand Ramanathan, ${ }^{1}$ Amelia Dobronyi, ${ }^{1}$ Xueyan Peng, ${ }^{2}$ Huanxing Sun, ${ }^{2}$ \\ Adrian Ledesma-Mendoza, ${ }^{1}$ Erica L. Herzog, ${ }^{2}$ and Anjelica L. Gonzalez ${ }^{1}$ \\ 'Department of Biomedical Engineering, Yale University, New Haven, Connecticut, USA. ${ }^{2}$ Section of Pulmonary, Critical \\ Care, and Sleep Medicine, Yale School of Medicine, Yale University, New Haven, Connecticut, USA.
}

\begin{abstract}
Idiopathic pulmonary fibrosis (IPF) is a fatal disease of unknown etiology characterized by a compositionally and mechanically altered extracellular matrix. Poor understanding of the origin of $\alpha$-smooth muscle actin ( $\alpha$-SMA) expressing myofibroblasts has hindered curative therapies. Though proposed as a source of myofibroblasts in mammalian tissues, identification of microvascular pericytes (PC) as contributors to $\alpha$-SMA-expressing populations in human IPF and the mechanisms driving this accumulation remain unexplored. Here, we demonstrate enhanced detection of $\alpha-S M A^{+}$cells coexpressing the PC marker neural/glial antigen 2 in the human IPF lung. Isolated human PC cultured on decellularized IPF lung matrices adopt expression of $\alpha$-SMA, demonstrating that these cells undergo phenotypic transition in response to direct contact with the extracellular matrix (ECM) of the fibrotic human lung. Using potentially novel human lung-conjugated hydrogels with tunable mechanical properties, we decoupled PC responses to matrix composition and stiffness to show that $\alpha$-SMA+ PC accumulate in a mechanosensitive manner independent of matrix composition. PC activated with TCF- $\beta 1$ remodel the normal lung matrix, increasing tissue stiffness to facilitate the emergence of $\alpha$-SMA+ PC via MKL-1/MTRFA mechanotranduction. Nintedanib, a tyrosine-kinase inhibitor approved for IPF treatment, restores the elastic modulus of fibrotic lung matrices to reverse the $\alpha-\mathrm{SMA}^{+}$phenotype. This work furthers our understanding of the role that microvascular PC play in the evolution of IPF, describes the creation of an ex vivo platform that advances the study of fibrosis, and presents a potentially novel mode of action for a commonly used antifibrotic therapy that has great relevance for human disease.
\end{abstract}

Conflict of interest: The authors have declared that no conflict of interest exists.

Submitted: July 17, 2017 Accepted: October 23, 2017 Published: December 7, 2017

Reference information: JCI Insight. 2017;2(23):e96352. https://doi.org/10.1172/jici. insight.96352.

\section{Introduction}

Idiopathic pulmonary fibrosis (IPF) is an incurable fibrosing condition for which there are no uniformly efficacious or curative therapies aside from lung transplantation (1-3). The disease is characterized by the replacement of functional lung tissue with a biochemically abnormal and stiffened extracellular matrix (ECM) that is produced by $\alpha$-smooth muscle actin-expressing ( $\alpha$-SMA-expressing) myofibroblasts. Myofibroblasts contribute to pathologic remodeling of the lung though their exertion of contractile forces, production of ECM, and response to TGF- $\beta 1$ (3). While the classical paradigm of fibrosis pathogenesis defines resident fibroblasts as the primary source of interstitial myofibroblasts, recent data suggest cells from the circulation, epithelium, endothelium, and adipose compartments may also contribute $(1,4)$. Emerging evidence implicates pericytes $(\mathrm{PC})$ as a source of myofibroblasts, contributing to matrix remodeling and vascular instability in other disease states (4-6). PC are microvascular mural cells of mesenchymal origin identified by their periendothelial location and their expression of neural/glial antigen (NG)2/chondroitin sulfate proteoglycan 4, platelet-derived growth factor receptor- $\beta$ (PDGFR- $\beta$ ), CD90, and CD146 (7, 8). PC respond to fibrotic stimuli by altering ECM deposition $(7,9,10)$, suggesting a potential role in liver, skin, kidney, and lung fibrosis $(4,11,12)$, and genetic fate mapping demonstrates that $\mathrm{PC}$ are a source of $\alpha$-SMA-expressing myofibroblasts in animal models of lung fibrosis (4). However, direct evidence linking PC to fibroproliferation and ECM remodeling that define IPF remains lacking.

Bioengineering-based strategies have emerged as useful tools to study cell-ECM interactions in several pathologies. Cells grown in decellularized matrices from healthy and diseased lungs can recapitulate 
salient mesenchymal, immunologic, and epithelial features of diseases like IPF (13), scleroderma (14), and chronic obstructive pulmonary disease (COPD) (15). However, the effect of human lung ECM on PC fibrosis-promoting behaviors remains unexplored. While it is increasingly recognized that mechanotransductive signaling and biochemical interactions can impact the differentiation and function of many cell lineages (16), currently used mimetic models of the lung microenvironment are unable to differentiate between these influences. Thus, the development of a humanized model decoupling tissue stiffness from matrix composition remains a critical unmet need in the study of pathologies in which both tissue mechanics and protein content are altered, such as IPF. With this in mind, we performed an expansive set of experiments aimed at understanding the contribution of PC to remodeling events that define IPF. Using both an engineered model and primary human lung tissues, we show that primary human PC adopt and retain myofibroblast properties in response to altered ECM compositions via a mechanosensitive process that is opposed by exposure to the tyrosine kinase inhibitor nintedanib.

\section{Results}

Myofibroblasts express PC markers in the IPF lung. To evaluate the contribution of $\mathrm{PC}$ to $\alpha$-SMA $\mathrm{SM}^{+}$populations in human IPF tissue, lung tissue obtained at autopsy from patients with IPF, or from control lungs from patients without known lung disease, underwent dual-color immunofluorescence for the myofibroblast marker $\alpha$-SMA and NG2, an integral membrane proteoglycan that identifies PC in the microvasculature (17). This approach identified a 5.8-fold increase in dual-positive cells in IPF lung tissue (Figure 1A), located in regions of parenchymal fibrosis, compared with controls, in which costaining was rare and restricted to the perivascular regions $(P<0.001$, Figure 1B). These data were confirmed by quantitative PCR-based (qPCR-based) evaluation of lung fibroblasts isolated from explant outgrowth from control and IPF lung tissue, where - in contrast to control cells - IPF fibroblasts displayed a 13-fold increase in NG2 expression $(P<0.001$, Figure 1C). When viewed in combination, these data support the contention that an NG2-expressing population, likely PC, contributes to the fibroblast accumulation in the IPF disease state.

a-SMA-expressing PC accumulate in response to IPF lung ECM. We next evaluated the potential of human $\mathrm{PC}$ to respond to the IPF lung microenvironment using 3-dimensional decellularized lung tissue, used to simulate the biochemical and biophysical properties of native lung tissue (18). Decellularization strategies have the advantage of removing cellular content of human lung (healthy and IPF) in its entirety, while maintaining presentation of major structural proteins $(19,20)$. However, small molecules and glycosaminoglycans are sacrificed in the stringent process of cell removal (21). Here, scaffolds prepared from decellularized IPF lung ECM were both compositionally and mechanically distinct from those of control lung (Supplemental Figure 1, A-C; supplemental material available online with this article; https://doi. org/10.1172/jci.insight.96352DS1), with the increased ECM density of IPF lungs resulting in an 11.6-fold increase in mechanical stiffness compared with control lungs at a physiological 20\% strain (Supplemental Figure 1, B and C). Because human lung PC isolates are difficult to obtain in sufficient numbers for experimentation and, when isolated, are collected from tissue resection of lung cancer patients (22-24), we made use of an alternate and inexhaustible source of PC: namely, the human placenta (25). We began by culturing NG2/CD90/PDGFR- $\beta$ /CD146-expressing human placental microvascular PC (Supplemental Figure 2) that were then seeded onto decellularized IPF or control lungs and cultured for 7 days. Similar to studies of lung-derived fibroblasts (2), IHC-based evaluation of $\alpha$-SMA revealed that, relative to cells grown on control samples, PC grown on decellularized IPF lungs increased expression of $\alpha$-SMA (Figure 2A), a finding that was confirmed by Western blotting $(P=0.006$, Figure $2 \mathrm{~B})$. These data show that human $\mathrm{PC}$ respond to factors present in the fibrotic lung microenvironment to engender a population of $\alpha-\mathrm{SMA}^{+}$cells.

Progressive matrix stiffening induces PC activation in normal human lung. We next sought to determine whether these observations resulted from biophysical or biochemical interactions with the fibrotic matrix. We conducted Western blot to quantify nuclear translocation of megakaryoblastic leukemia 1 (MKL-1), also known as myocardin-related transcription factor A (MRTF-A). MKL-1 is a key transcription factor implicated in cellular responses to local stiffness (26). Nuclear translocation of MKL-1 was increased by 1.95 -fold in PC cultured in IPF lung versus healthy lung $(P=0.048$, Figure $2 C)$, suggestive of active mechanosensing. To further study this process, we developed a culture platform that could provide the unique opportunity to decouple ECM stiffness and composition in the ex vivo setting. Here, polyacrylamide hydrogels of low $(1.8 \pm 0.5 \mathrm{kPa})$, medium $(4.4 \pm 0.5 \mathrm{kPa})$, and high $(23.7 \pm 2.3 \mathrm{kPa})$ stiffness were generated to approximate the healthy, transitioning, and fibrotic human lung, respectively (Figure 3, A 


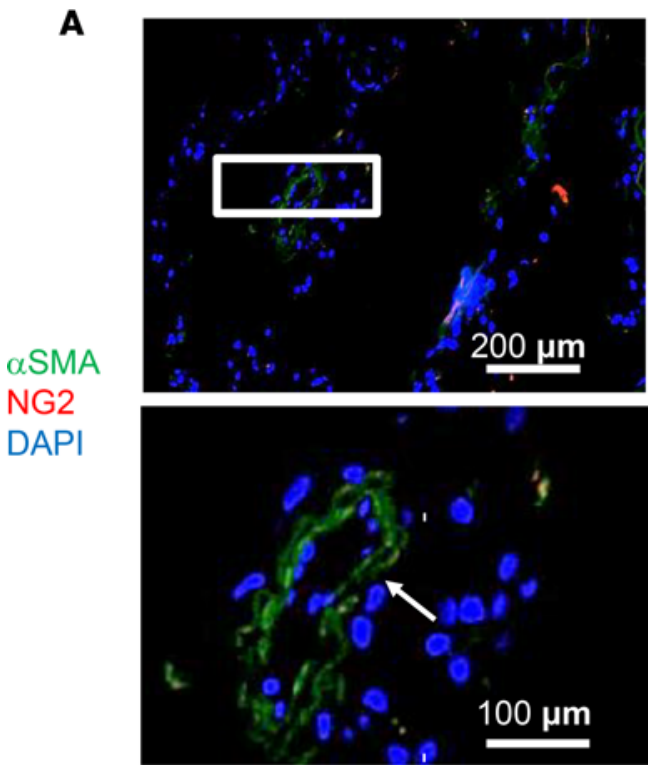

Control

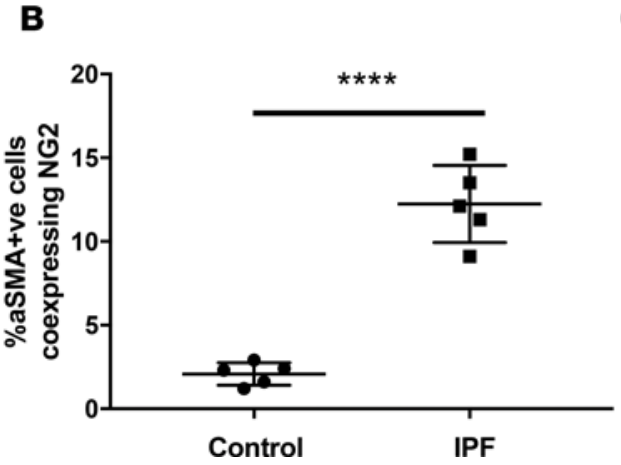

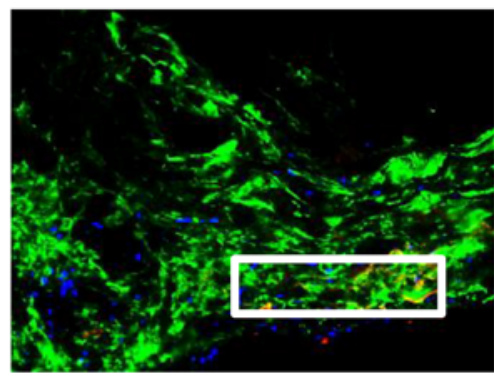

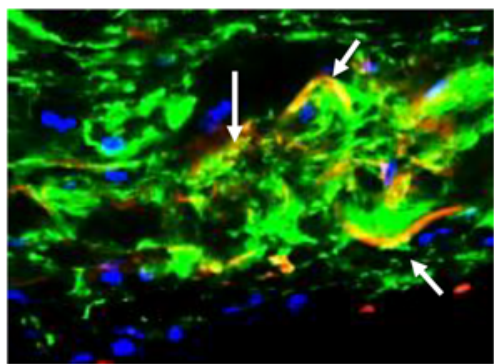

IPF

C

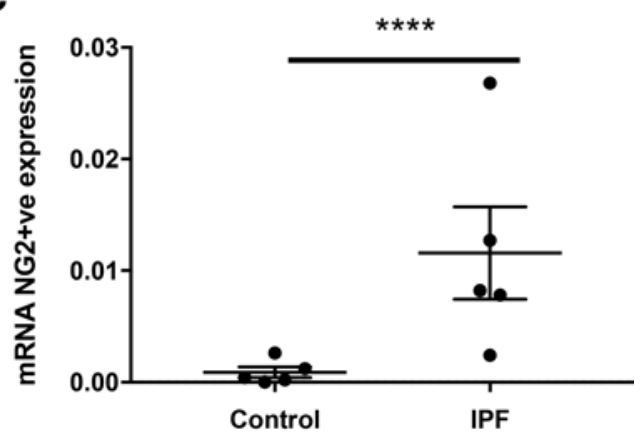

Figure 1. $\alpha$-SMA+ pericyte (PC) expression accumulate in fibrotic foci of idiopathic pulmonary fibrosis (IPF) human lungs. Control and IPF lung samples were fixed and stained to observe contribution of PC to myofibroblast population in IPF. (A) Immunofluorescence images of human lung tissue from control and IPF patients. Human lungs are costained for $\alpha$-smooth muscle actin ( $\alpha$-SMA, green) with PC marker NG2 (red) and Dapi (blue). Boxes and arrows represent areas of NG2 and $\alpha$-SMA dual positive costaining in lung tissue. (B) Image analysis used to quantify the \% coexpression \pm SEM of $\alpha-\mathrm{SMA}^{+} \mathrm{NC2} 2^{+}$cells in control and IPF lung samples. (C) Compared with NHLFs, relative expression of NC2 message is increased in IPF lung fibroblasts (2-tailed Student $t$ test with Bonferroni post-test, ${ }^{* * *} P<0.001$ compared with control lung, $n=5$ ).

and B, and Supplemental Figure 3) (27). These gels were then functionalized through chemical conjugation of solubilized ECM from control or IPF lung (Supplemental Figure 3). When PC seeded onto these substrates were analyzed after 7 days of culture (Figure $3 \mathrm{C}$ ), substrate stiffness was found to be the dominant driver of myofibroblastic properties. Specifically, relative to cells grown on the low-stiffness hydrogels, PC cultured on intermediate- and high-stiffness hydrogels displayed significantly increased cell area (Figure 3D), elongation (Figure 3E), and expression of $\alpha$-SMA (Figure 3F) in a manner that was independent of the source of conjugated ECM (control or IPF). Neither collagen I nor EDA fibronectin, both of which have been reported to induce $\alpha$-SMA in fibroblasts (2), were capable of overcoming the effect of reduced matrix elasticity in our model (Supplemental Figure 4). Interestingly, there was no significant difference in $\alpha$-SMA expression between cells cultured on intermediate- and high-stiffness hydrogels (Figure 3F and Supplemental Figure 4). In further support of a mechanosensitive mechanism, nuclear translocation of MKL1 increased when PC were cultured on substrates of increasing stiffness, including both medium- $(P=0.046)$ and high-stiffness $(P=0.008)$ hydrogels (Figure $3, \mathrm{G}$ and $\mathrm{H})$. Furthermore, treatment with the MKL-1 inhibitor CCG-1423 at day $0(P<0.001)$ or day $7(P=0.039)$ of PC culture significantly reduced the detectable quantities of $\alpha$-SMA (Figure 3, I and J). These data indicate that even a small increase in substrate stiffness is sufficient to promote myofibroblast-like characteristics in human PC via mechanotransductive signaling. 
A a-SMA
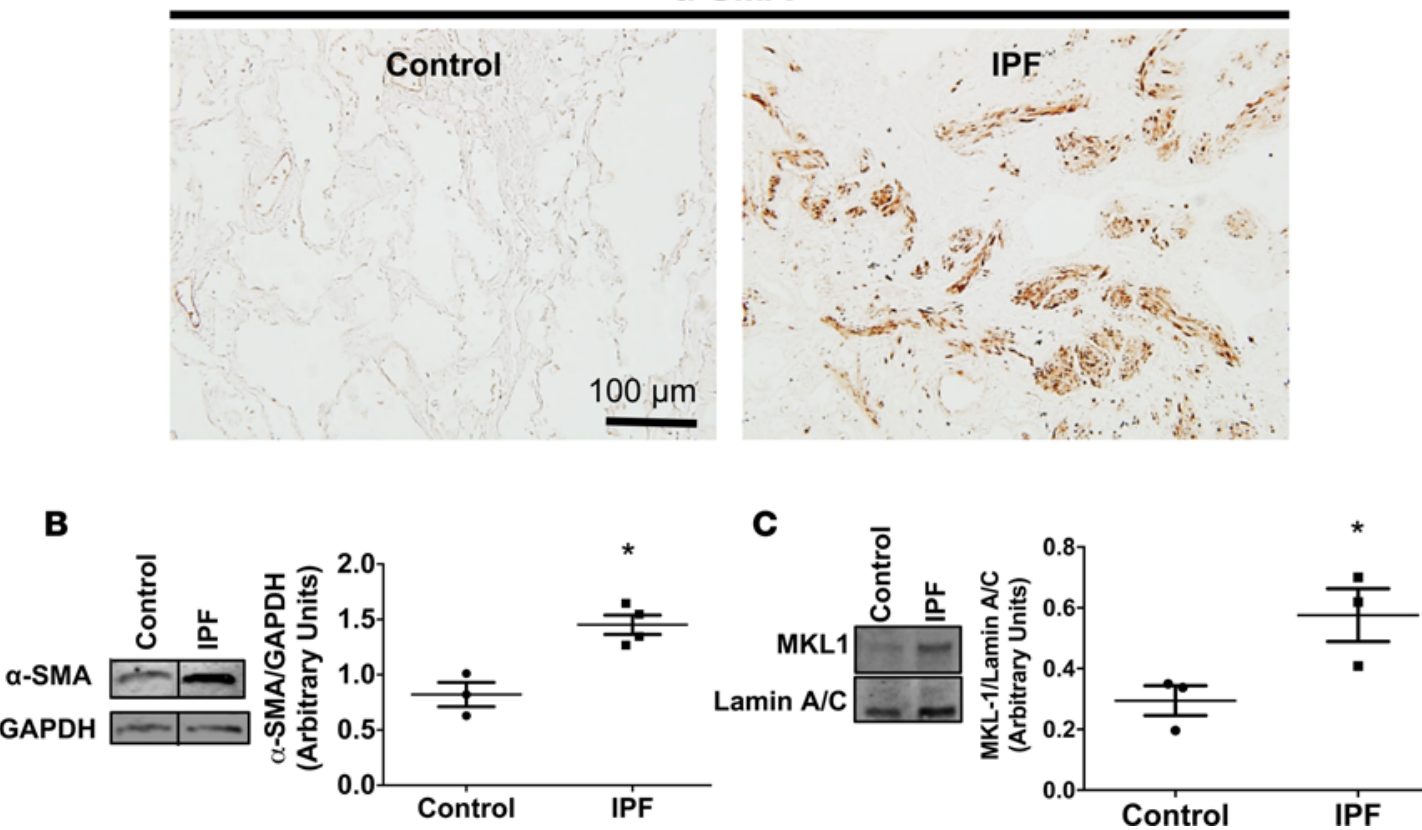

Figure 2. Human idiopathic pulmonary fibrosis (IPF) lung extracellular matrix (ECM) facilitates mechanoresponsive expression of $\alpha$-SMA. (A) Seven-day PC cultures on decellularized lung scaffolds were stained for $\alpha$-SMA and IHC on control and IPF ECM. (B) $\alpha$-SMA expression presented as mean \pm SEM using immunoblotting and normalized to GAPDH (black lines used to designate samples run in the same gel but noncontiguous lanes). (C) MKL1 translocation was examined by extracting nuclear protein for PC cultured on control or IPF lung, was analyzed using immunoblotting, and was presented as mean \pm SEM normalized to lamin A/C (2-tailed Student $t$ test with Bonferroni post-test, ${ }^{*} P<0.05$ compared with control lung, $n=3$ and 4 ).

Exposure to exogenous TGF- $\beta 1$ does not influence accumulation of a-SMA+PC. TGF- $\beta 1$ acts as a central mediator of fibrosis that regulates the fibroblast-to-myofibroblast transition, though its role in mechanotransductive processes has been incompletely explored. To determine whether exposure to TGF- $\beta 1$ affects expression of $\alpha$-SMA, freshly isolated PC were grown on soft or stiff hydrogels for up to 7 days in the presence or absence of TGF- $\beta 1$. Interestingly, activation with TGF- $\beta 1$ failed to affect the proportion of $\alpha$-SMA-expressing PC cultured on hydrogels of any stiffness (Figure $4, \mathrm{~A}$ and B). These data indicate that, unlike fibroblasts (28), short-term exposure to TGF- $\beta 1$ is insufficient to oppose the effects of substrate stiffness, further supporting a central role for mechanosensing in the initial accumulation of $\alpha$-SMA-expressing PC.

TGF- $\beta 1$ activation induces $P C$ matrix deposition. Current paradigms of lung fibrosis propose that the TGF- $\beta 1$-rich milieu occurring in the later stages of fibrosis favors the accumulation and activation of matrix-producing myofibroblasts (1). The bioinert and hydrophilic nature of our hydrogel does not allow for examination of PC matrix deposition. Therefore, we developed a system in which PC grown on glass allows accumulation and evaluation of deposited ECM. The PC were exposed to TGF- $\beta 1$ for up to 28 days as a model of the chronic exposure to fibrogenic stimuli that would be encountered in the fibrotic human lung (Figure 4C). In this context, an alternate effect of TGF- $\beta 1$ might involve a potentially novel and previously unrecognized autocrine contribution to ECM deposition by microvascular cells. This prediction proved accurate, as - following long-term activation - PC responded to TGF- $\beta 1$ by producing circumferential nodes of ECM consisting of a central nucleus high in collagen I and collagen IV, surrounded by a radiating matrix that was rich in fibronectin (Figure $4 \mathrm{C}$ ). Node formation was first detected after 7 days of TGF- $\beta 1$ exposure, with a 2.2 -fold $(P<0.001)$ and 1.8 fold $(P<0.01)$ increase in the formation of lesions at days 14 and 28 , respectively, compared with the nonactivated control (Figure 4, C-E). The number of nodes following TGF- $\beta 1$ activation peaked at day 14 and decreased by day 28, likely due to a ripening effect wherein individual foci coalesce to become larger, singular foci (Figure 4E). Immunofluorescence evaluation revealed that cells within the nodes expressed high levels of $\alpha$-SMA and vimentin compared with the surrounding cells (Figure $4 \mathrm{~F}$ ). Interestingly, in this model, TGF- $\beta 1$-activated PC exhibited a significant increase in both $\alpha$-SMA $(P=$ 
A

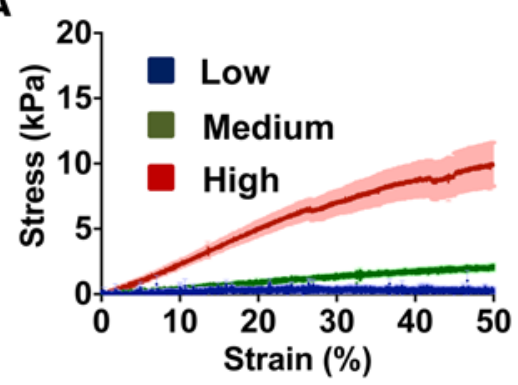

B

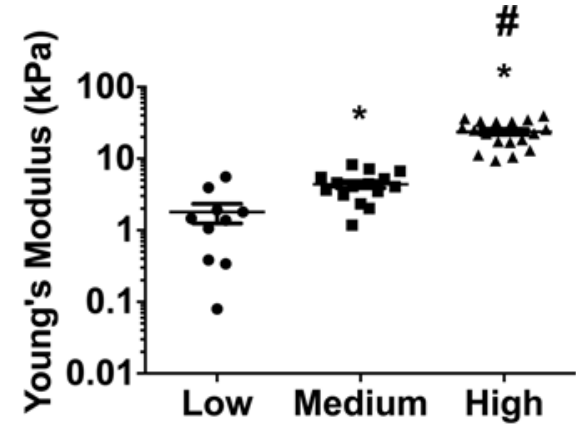

C
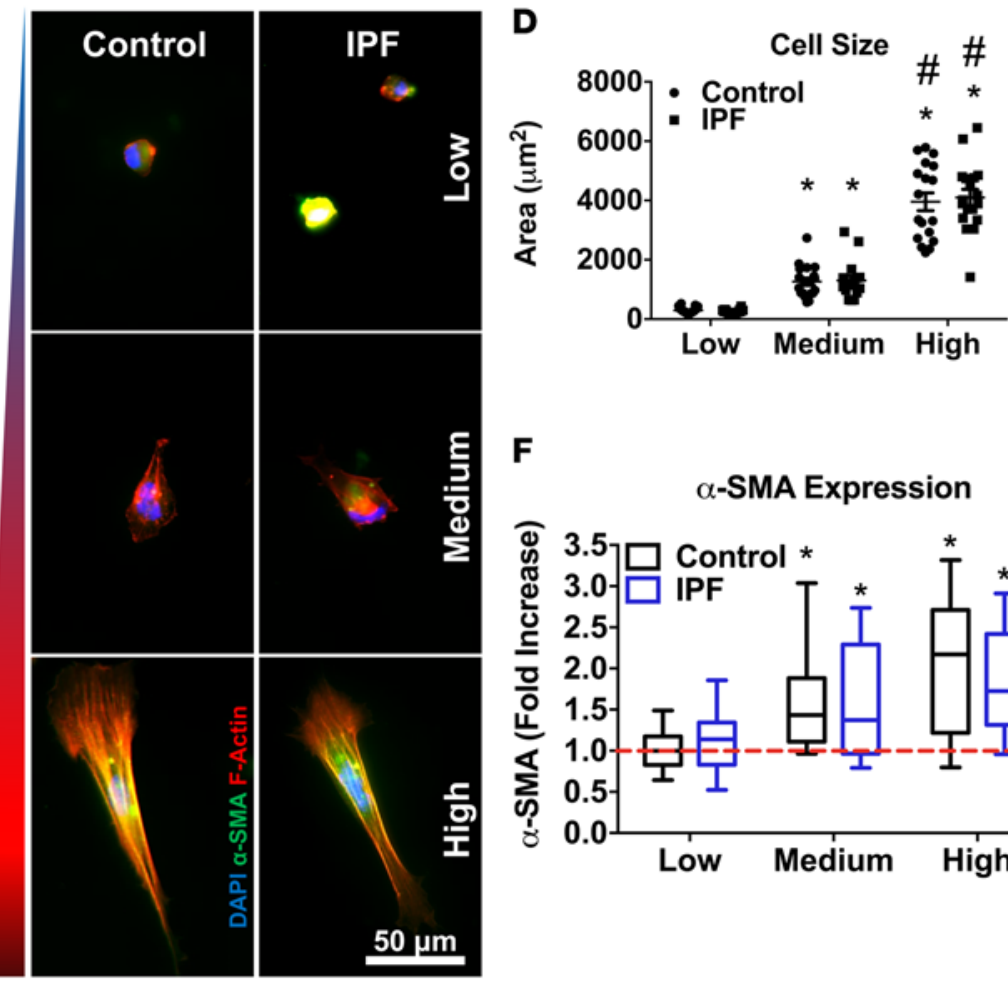

E

Cell Shape

$\mathbf{F}$

$\alpha$-SMA Expression

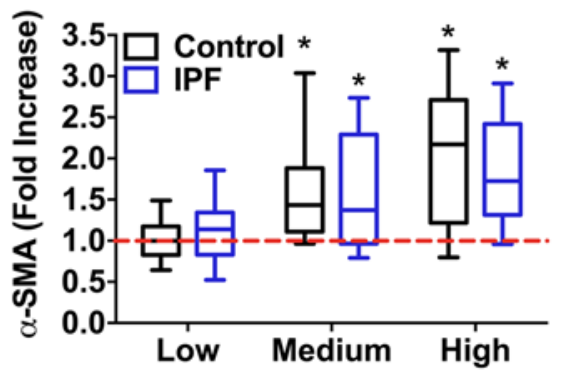

G
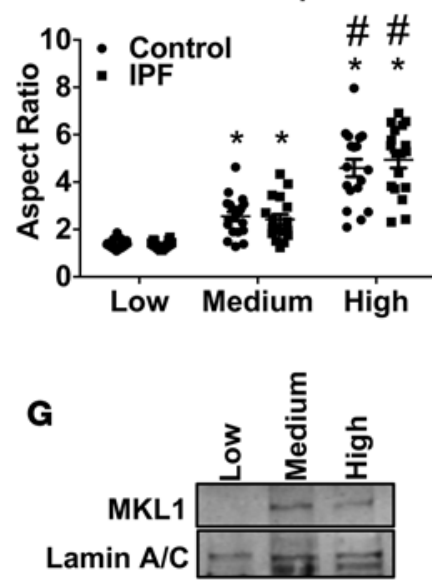

H

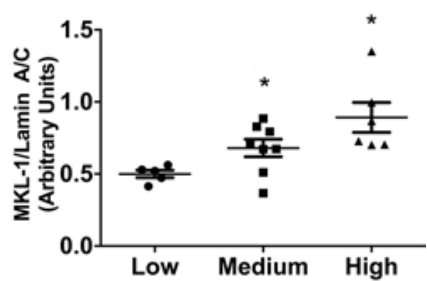

I
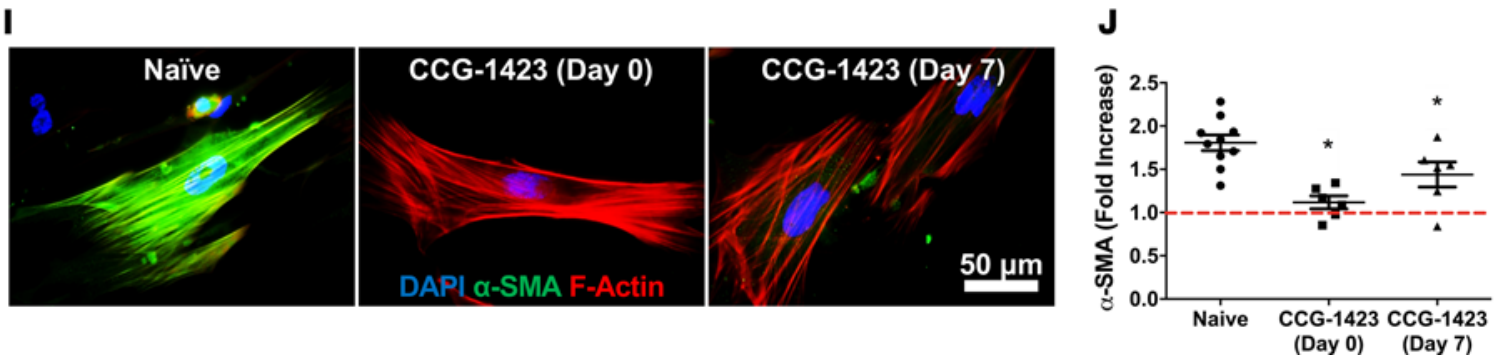

Figure 3. Increased substrate stiffness induces megakaryoblastic leukemia 1-dependent (MKL1-dependent) $\alpha$-SMA expression. Round glass coverslips were coated with polyacrylamide hydrogels with varying stiffness to evaluate the mechanotransductive effects in pericytes. Elasticity of polyacrylamide hydrogels was tested to generate (A) stress-strain plots and (B) Young's Moduli calculated at 20\% strain, reported as mean \pm SEM (one-way ANOVA with Tukey Post-Hoc test, ${ }^{*} P<0.05$ versus low-stiffness hydrogel, ${ }^{\#} P<0.05$ versus medium stiffness hydrogel, $n=9$ and 10 ). Control and IPF lung protein were conjugated to the polymer, and PC were cultured for 7 days. (C) Immunofluorescence images of $\alpha$-SMA (green) and F-Actin (red) were used to determine (D) cell area \pm SEM and (E) shape (aspect ratio \pm SEM) (one-way ANOVA with Tukey Post-Hoc test, ${ }^{*} P<0.05$ compared with low-stiffness hydrogel of the same matrix, ${ }^{\#} P<0.05$ versus medium stiffness hydrogel of the same matrix, $n \geq 10$ ). (F) $\alpha$-SMA expression was quantified using flow cytometry, presented as mean fold increase over expression of cells on low-stiffness healthy lung \pm SEM (one-way ANOVA with Tukey Post-Hoc test $* P<0.05$ compared with low-stiffness hydrogel of the same matrix, $n=9$ ). (G) MKL1 translocation was analyzed using immunoblotting and $(\mathbf{H})$ reported as mean \pm SEM normalized to lamin A/C (one-way ANOVA with Tukey Post-Hoc test, ${ }^{*} P<0.05$ versus low-stiffness hydrogel, $n=5$ ). (I) Immunofluorescence images of $\alpha$-SMA (green), F-Actin (red), and nuclei (blue) are shown for PC on high-stiffness hydrogels with no treatment (naive) and 0- or 7-day treatment with CCG-1423, an inhibitor of MKL1. (J) $\alpha$-SMA expression collected cells was quantified using flow cytometry and presented as fold increase in $\alpha$-SMA \pm SEM compared with untreated cells on low-stiffness healthy lung (one-way ANOVA with Tukey Post-Hoc test, ${ }^{*} P<0.05$ versus naive PC, $n=4-6$ ). 
0.011) and vimentin $(P<0.0001)$ expression compared with the nonactivated control (Figure $4, \mathrm{~F}-\mathrm{I})$. These data indicate that PC respond to TGF- $\beta 1$ by adopting functional characteristics of myofibroblasts, including the ability to produce a collagen and fibronectin-rich ECM, which in turn facilitates the increased expression of $\alpha$-SMA and vimentin by these highly plastic cells.

$T G F-\beta 1-i n d u c e d$ stimulation of PC matrix production is opposed by treatment with nintedanib. Currently available IPF therapeutics, including the small molecule pyridone derivative pirfenidone and the tyrosine-kinase inhibitor nintedanib, can delay but not prevent or reverse the progression of fibrosis (29). Nintedanib is known to oppose TGF- $\beta 1$-induced myofibroblast differentiation and matrix deposition of cultured lung fibroblasts in vitro (30), but its effect on these endpoints in PC has not been defined. In order to address this issue, $\alpha-\mathrm{SMA}^{+}$, vimentin ${ }^{+}$, and matrix-producing PC were generated via long-term exposure to TGF- $\beta 1$ as described above, and they were subsequently treated with TGF- $\beta 1$ with or without nintedanib for an additional 7 days. Importantly, nintedanib substantially reduced detection of ECM components (Figure 5, A-E) in a manner that was independent of effects on $\alpha$-SMA or vimentin (Figure 5, F-H). Specifically, nintedanib treatment reduced collagen I $(P=0.01)$ and fibronectin $(P=0.002)$ fibrotic node formation compared with the increase observed following TGF- $\beta 1$ activation of PC $(P=0.049$, Figure $5, \mathrm{~A}$ and $\mathrm{E})$ without affecting the expression of $\alpha$-SMA or vimentin (Figure $5, \mathrm{~F}$ and $\mathrm{G})$. These data demonstrate a potentially novel and previously unrecognized mechanism of action of nintedanib that involves the regulation of ECM production by PC-derived myofibroblasts.

$T G F-\beta 1-$ induced lung remodeling properties of a-SMA+ $P C$ are reduced by nintedanib. The studies described above present compelling evidence of the ability of PC to modify and respond to their local microenvironment. However, because these studies were performed on glass substrates, their relevance to the human lung remains uncertain. We therefore evaluated the ability of PC to cause pathologic remodeling and ECM accumulation in a more realistic culture system: namely, the decellularized human lung. PC were seeded on scaffolds prepared from decellularized healthy lungs, activated with TGF- $\beta 1$ for 21 days, and further stimulated cells with TGF- $\beta 1$ in the presence or absence of nintedanib. Masson's trichrome and Picosirius red staining revealed that treatment of PC with 28 days of TGF- $\beta 1$ increased both ECM density and collagen I crosslinking (Figure 5, I and J). These findings were accompanied by an 8.6-fold increase in the elastic modulus of the scaffold to levels similar to those of IPF lungs ( $P=0.001$, Figure 5J). Importantly, in this model, treatment with nintedanib reduced the number of PC expressing $\alpha$-SMA by $90.1 \%(P<0.0001$, Figure $5, \mathrm{~K}$ and $\mathrm{L})$ in a manner that was accompanied by a reduction in both matrix density and collagen I content (Figure 5I). Treatment with nintedanib also caused reversion of the elastic modulus to baseline levels $(P=0.44$ compared with control lung; $P=0.001$ compared with TGF- $\beta 1$ without nintedanib-treated lung) (Figure 5J). These data show that TGF- $\beta 1$-stimulated PC can remodel otherwise normal lung ECM and that these changes are opposed by treatment with nintedanib.

Mechanism of nintedanib therapeutic function in PC-induced lung fibrosis. The mechanism of nintedanib efficacy is incompletely defined and, thus far, has ignored any effects on PC. Because treatment with nintedanib was able to reduce the stiffness of fibrotic lung matrices, we reasoned that its mechanism of action in this context must involve degenerative remodeling of the ECM, perhaps through the secretion and activation of matrix metalloproteinases (MMPs). Thus, supernatants recovered from TGF- $\beta 1$-activated PC were subject to ELISA-based detection of MMP8, -9, and -13, which revealed substantial increases in all 3 mediators following nintedanib treatment $(P<0.05, P<0.01$, and $P<0.001$, respectively, compared with treatment with TGF- $\beta 1$ alone; Figure 6, A-D). Nintedanib treatment did not increase expression of MMP10 (Figure 6E). Finally, generation of active collagenases MMP2 and MMP9 was also enhanced when TGF- $\beta 1$-activated PC were treated with nintedanib ( $P<0.05$ and $P<0.01$, respectively; Figure $6, \mathrm{~F}$ and $\mathrm{G}$ ), providing evidence for the matrix remodeling and increased lung tissue elasticity previously observed. Together, these data demonstrate the unexpected ability of PC to respond to and modify their microenvironment, demonstrating both the regulated production of ECM and the generation of matrix-degrading MMPs in response to exogenous stimuli. They also extend the mode of action of IPF therapies to include the regulation of PC-mediated matrix remodeling events via a mechanism involving production and activation of MMPs.

\section{Discussion}

These data provide important new insight into the role of PC in the development and perpetuation of fibrosis and remodeling of the human lung. A subpopulation of $\alpha$-SMA-expressing IPF lung myofibroblasts express PC markers, thereby showing, for the first time to our knowledge, a contribution of PC to fibroproliferative lung disease in humans. In an ex vivo corollary of these findings, we used an experimental platform based on primary human cells and tissues to decouple the biochemical and biomechanical regulation of PC phenotype. 
A

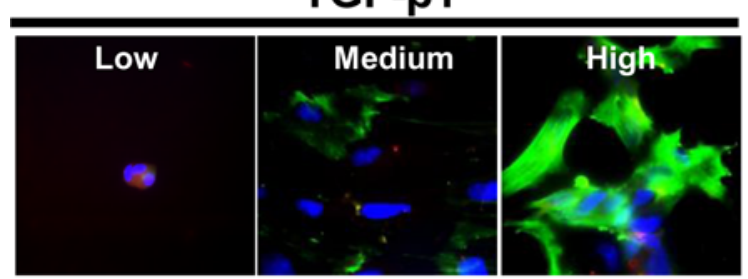

aSMA Vimentin DAPI
B

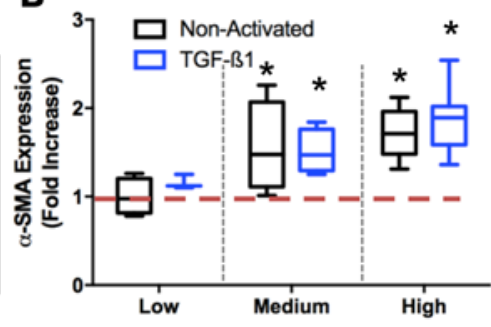

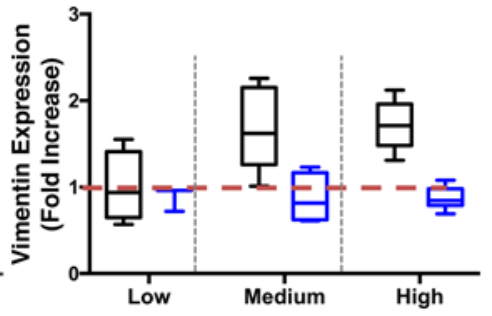

C
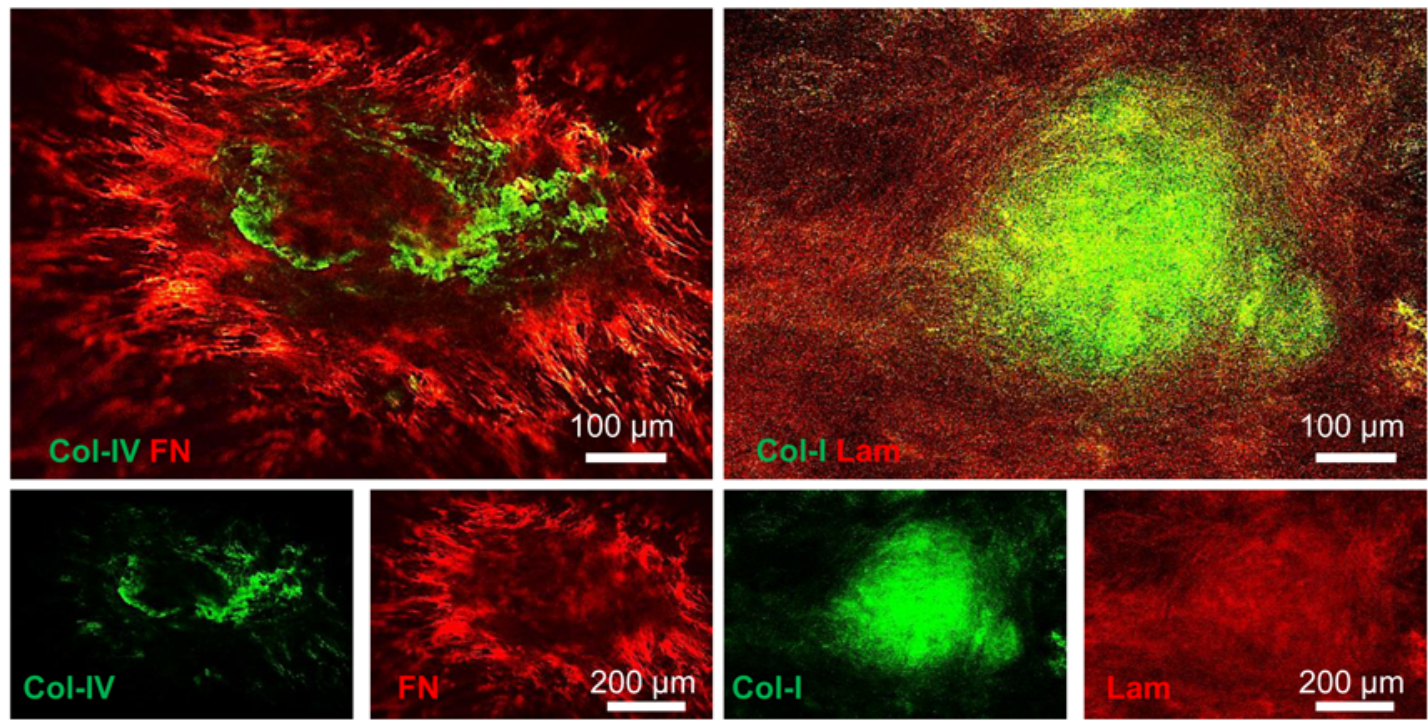

D

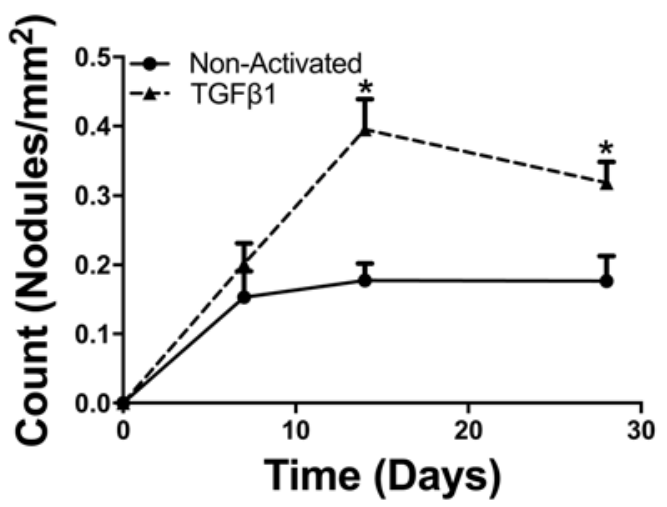

E

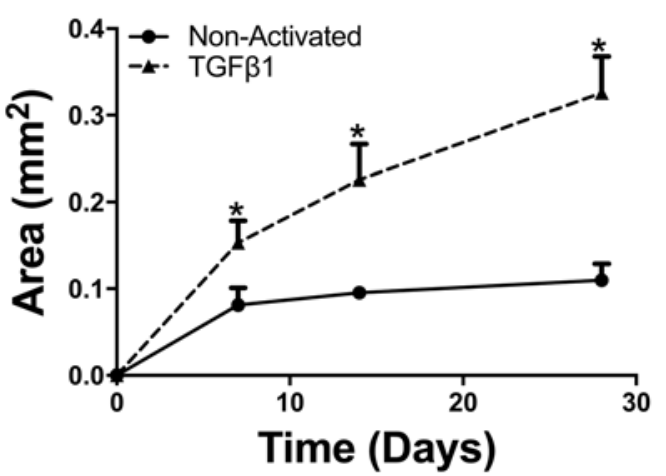

$\mathbf{F}$

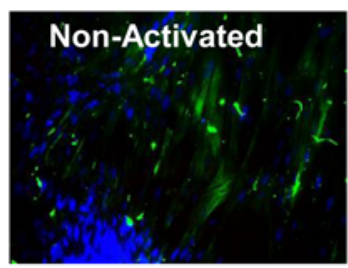

aSMA Vimentin DAPI

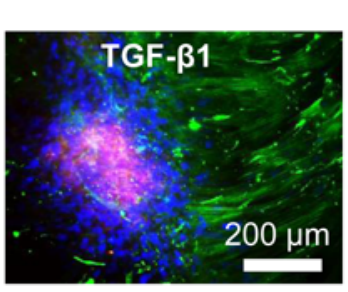

G

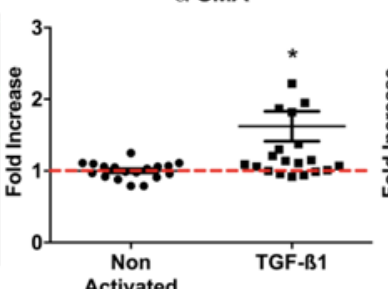

H

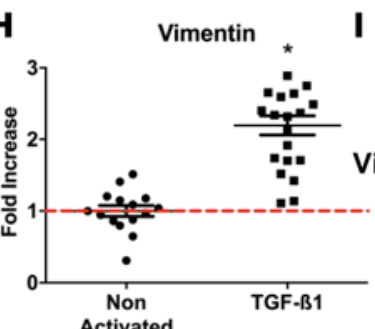

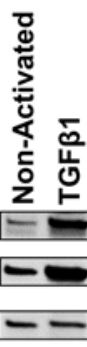

GAPDH --

Figure 4. TGF- $\beta 1$ promotes extracellular matrix (ECM) remodeling and fibrotic foci formation. (A) PC were cultured on matrices and activated with TCF- $\beta 1$ for 14 days. Immunofluorescence images of $\alpha$-SMA (green), vimentin (red), and nuclei (blue) are shown and mean expression \pm SEM. (B) $\alpha$-SMA and vimentin were quantified using flow cytometry (one-way ANOVA with Tukey Post-Hoc test, ${ }^{*} P<0.05$ compared with low-stiffness hydrogel of the same activation, ${ }^{\#} P<0.05$ compared with nonactivated condition of the same hydrogel stiffness, $n=3$ ). (C) Immunofluorescence images of collagen IV (green) and fibronectin (red), or collagen I (green) and laminin (red), of fibrotic lesions are shown, and (D) the mean spatial density \pm SEM and (E) mean area \pm SEM of the lesions were quantified (one-way ANOVA with Tukey Post-Hoc test, ${ }^{*} P<0.05$ versus nonactivated PC, $n=6$ ). (F) Immunofluorescence images of $\alpha$-SMA and vimentin. (G) $\alpha$-SMA and (H) vimentin expression quantified using flow cytometry (mean fold increase over nonactivated $\alpha$-SMA or vimentin \pm SEM shown) and (I) confirmed using immunoblotting (Student $t$ test with Bonferroni post-test. ${ }^{*} P<0.05$ versus nonactivated PC, $n \geq 10$ ). 
A

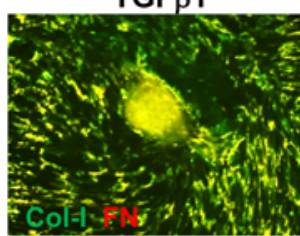

E

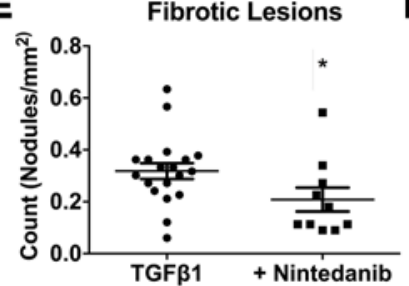

I

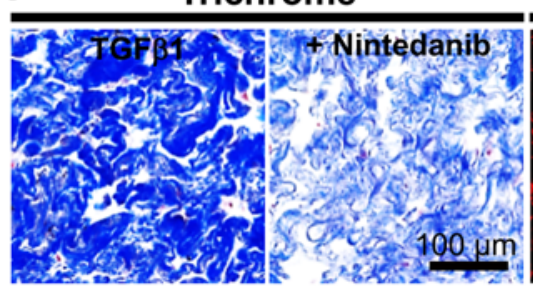

+Nintedanib

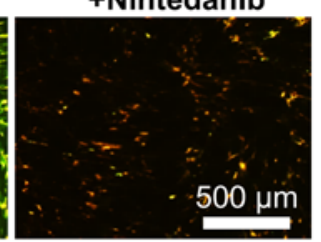

$\mathbf{F}$

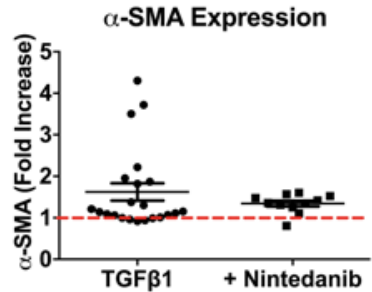

B

FN

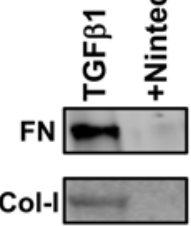

Collagen I

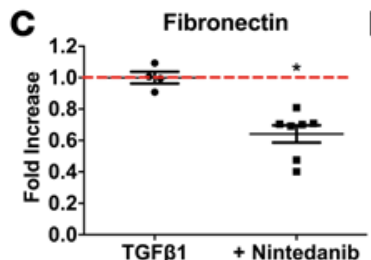

G
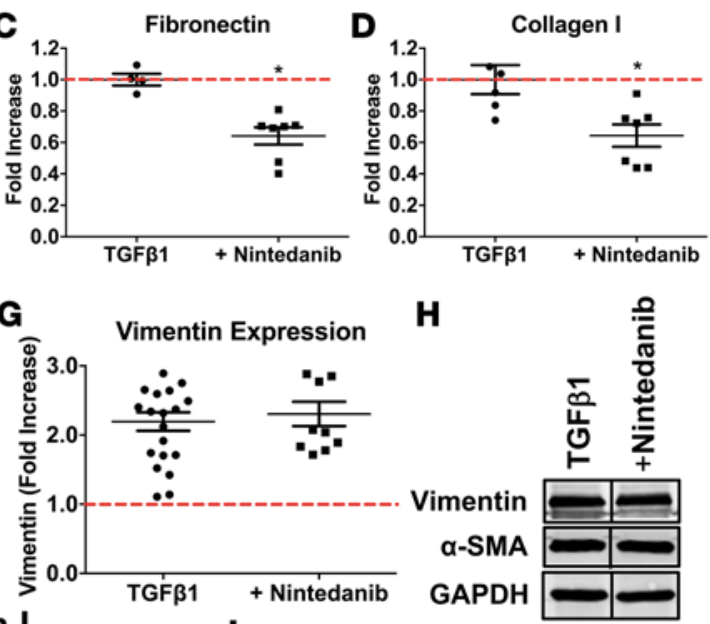

H

K

a-SMA

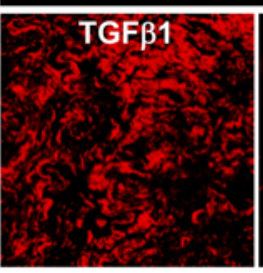

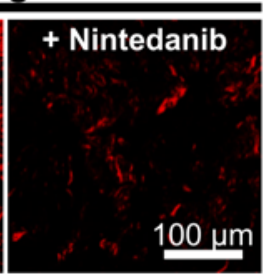

\section{$J$}

(a)

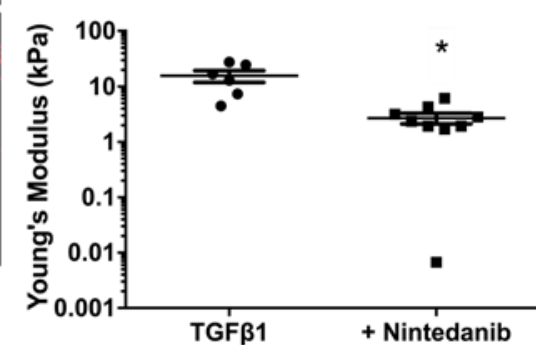

$\mathbf{L}$

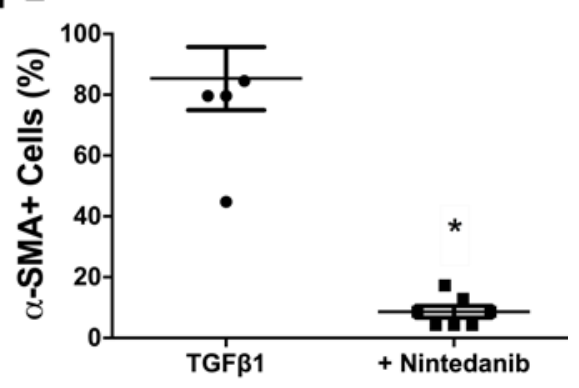

Figure 5. Effects of TGF- $\beta 1$ induced lung matrix stiffening and increased $\alpha$-SMA expression are reversed by nintedanib treatment. PC were cultured on glass, activated with TCF- $\beta 1$ for 28 days, and treated with nintedanib for 7 days. (A) Immunofluorescence images of collagen I (green) and fibronectin (red). (B-D) Matrix deposition was quantified using immunoblotting $(n=7)$, and (E) fibrotic lesions were quantified from images $(n \geq 10)$ at day 28 (represented as mean \pm SEM, Student $t$ test with Bonferroni post-test, ${ }^{*} P<0.05$ versus TGF- $\left.\beta 1\right)$. (F) $\alpha$-SMA and (G) vimentin expression, quantified using flow cytometry (presented as mean fold increase over protein expression of cells cultured on low-stiffness healthy lung \pm SEM) (H) were confirmed using immunoblotting ( $n \geq 10$ ). Black lines used to designate samples run in same gel but in noncontiguous lanes. (I) PC were cultured on decellularized control lung with TGF- $\beta 1$ activation for 28 days and treated with nintedanib for 7 days. Matrix deposition was evaluated by Masson's trichrome and Picrosirius red staining. (J) Mean Young's Moduli \pm SEM of the decellularized lung were determined using an Instron5848 at $20 \%$ strain (Student $t$ test with Bonferroni post-test ${ }^{*} P<0.05$ versus TCF- $\beta 1, n$

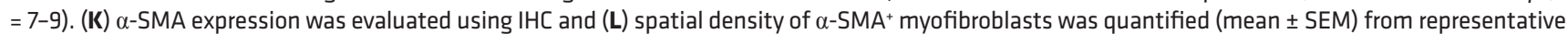
$\alpha$-SMA IHC images (Student $t$ test with Bonferroni post-test, ${ }^{*} P<0.05$ versus TCF- $\beta 1, n=4-6$ ).

The accumulation of $\alpha$-SMA-expressing PC was driven by contact with the diseased ECM in an MKL1-dependent mechanotransductive process that is largely independent of ECM composition. Moreover, this study aims to highlight the importance of mechanosensing pathways for the accumulation of $\alpha-\mathrm{SMA}^{+} \mathrm{PC}$ in the fibrotic foci. However, these pathways, while well studied in fibroblasts, remain to be confirmed in PC. For example, focal adhesions and focal adhesion kinases (FAK) play an integral part of $\alpha$-SMA expression in fibroblast-to-myofibroblast transdifferentiation (31) as a means of signal transduction from clustered integrin-ligand binding interactions between cells and the fibrotic ECM. Additionally, immunostaining of fibroblasts grown on fibronectin-coated gels with varying stiffness revealed an increase in nuclear translocation of yes-associated protein (YAP) and transcriptional coactivator with PDZ-binding motif (TAZ) in fibroblasts seeded on stiffer substrates (32). A YAP/TAZ-dependent mechanism in PC following TGF- $\beta 1$ activation has yet to be deter- 


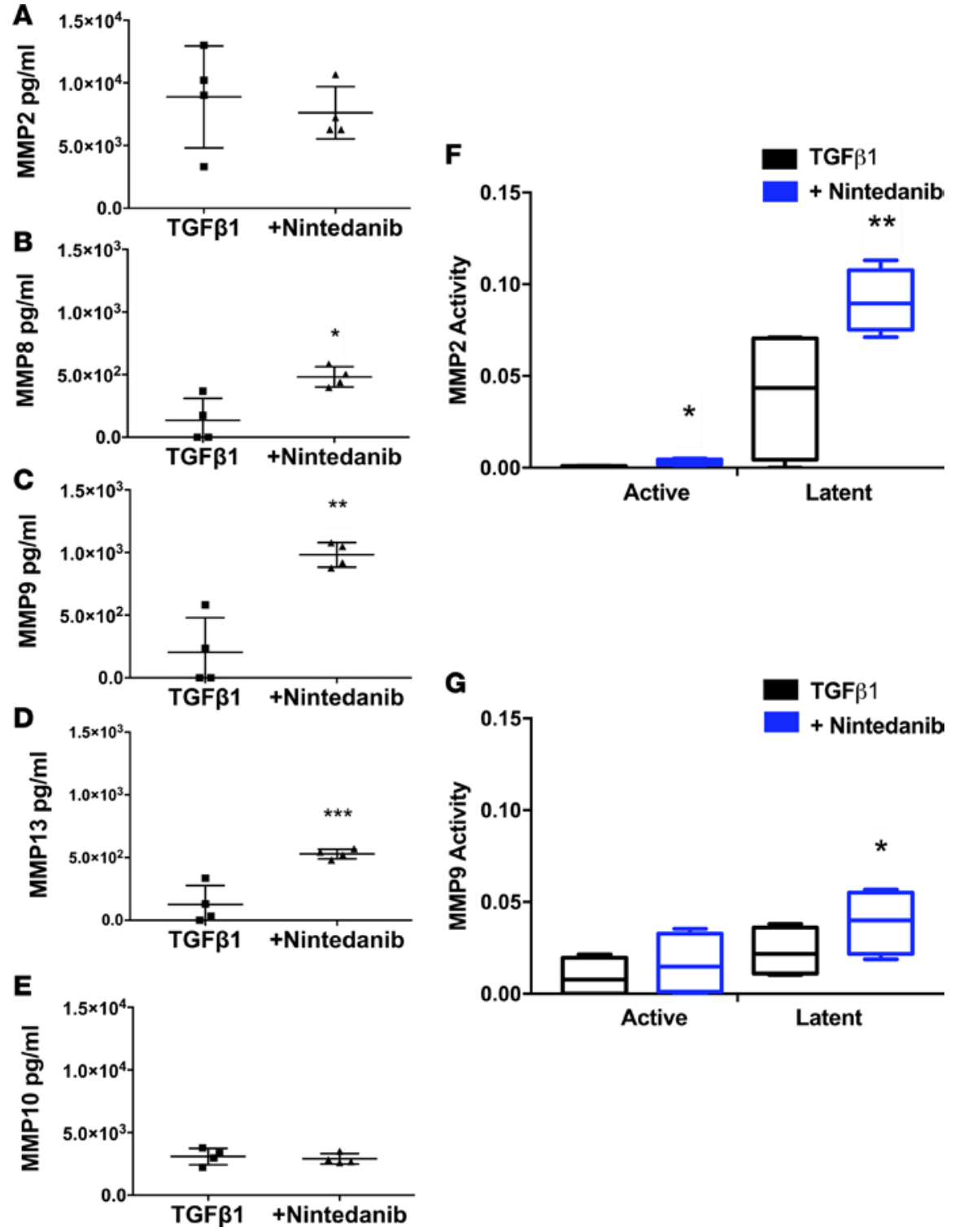

Figure 6. Nintedanib induces elevated pericyte (PC) production of metalloproteinases MMP8, MMP9, and MMP13. (A-E) PC supernatant activated with TGF- $\beta 1 \pm$ nintedanib were evaluated using ELISA against (A) MMP2, (B) MMP8, (C) MMP9, (D) MMP13, and (E) MMP10 and data presented as detected MMP concentration (pg/ $\mathrm{ml}) \pm \mathrm{SEM}$ (Student $t$ test with Bonferroni posttest; ${ }^{*} P<0.05$, ${ }^{* *} P<0.01$ versus TGF- $\beta 1 ; n=4$, ${ }^{* * *} P<0.001$ ). (F and $\mathbf{G}$ ) Gelatinase zymography was also used to validate activity of (F) MMP2 and (G) MMP9 production by PC \pm nintedanib treatment (one-way ANOVA with Tukey post-hoc test, ${ }^{*} P<0.05$ versus TCF- $\beta 1, n=6 ;{ }^{* *} P<0.01$ ).

mined (Figure 7). While short-term treatment of PC with TGF- $\beta 1$ does not alter $\alpha$-SMA expression, longer-term TGF- $\beta 1$ exposure expands the $\alpha$-SMA ${ }^{+}$, vimentin ${ }^{+}$population capable of producing excess ECM. These cells also remodeled decellularized normal lung scaffolds through collagen and fibronectin deposition and enhancement of matrix stiffness. This effect is reversible through treatment with the tyrosine-kinase inhibitor nintedanib via production of MMPs. Altogether, these data support the contention that PC-derived myofibroblasts arise in response to altered tissue stiffness to contribute to lung remodeling and ECM accumulation in IPF.

Our ex vivo analysis shows that local substrate stiffness regulates the early events in accumulation of $\alpha-\mathrm{SMA}^{+} \mathrm{PC}$, as cells seeded into the decellularized IPF lung show increased engraftment and persistence of $\alpha-\mathrm{SMA}^{+}$cells compared with those seeded into control lung scaffolds. Interestingly, these events are regulated by cellular responses to local substrate stiffness and not solely as an effect of receptor-ligand interactions with specific biochemical components of the IPF ECM. We observe that even small increases in the elastic modulus are sufficient to drive the $\alpha$-SMA ${ }^{+}$phenotype, suggesting that the severity of matrix stiffening seen in the end-stage IPF lung is not necessary to drive PC accumulation. Since stiffness, and not matrix composition or TGF- $\beta 1$, drove the initial accumulation of $\alpha-\mathrm{SMA}^{+} \mathrm{PC}$, these findings suggest that any condition that increases the elastic modulus - such as other forms of fibrotic lung disease, acute respiratory distress syndrome (ARDS), occupational lung disease, ventilator induced lung injury, and even normal aging - might be sufficient to expand PC in this manner. 
A Human Lung

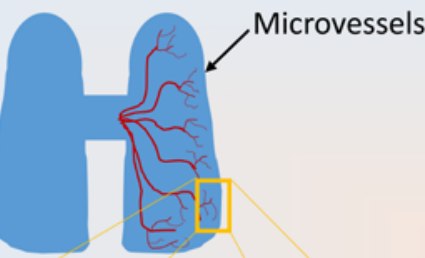

B

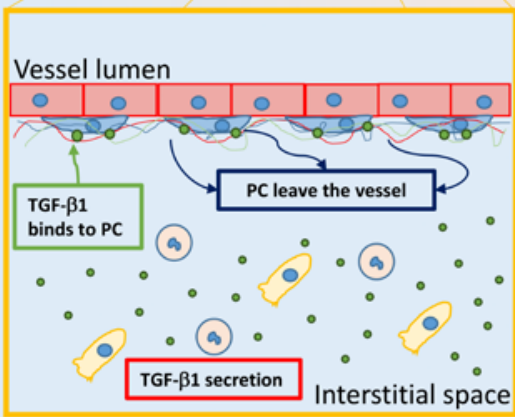

C

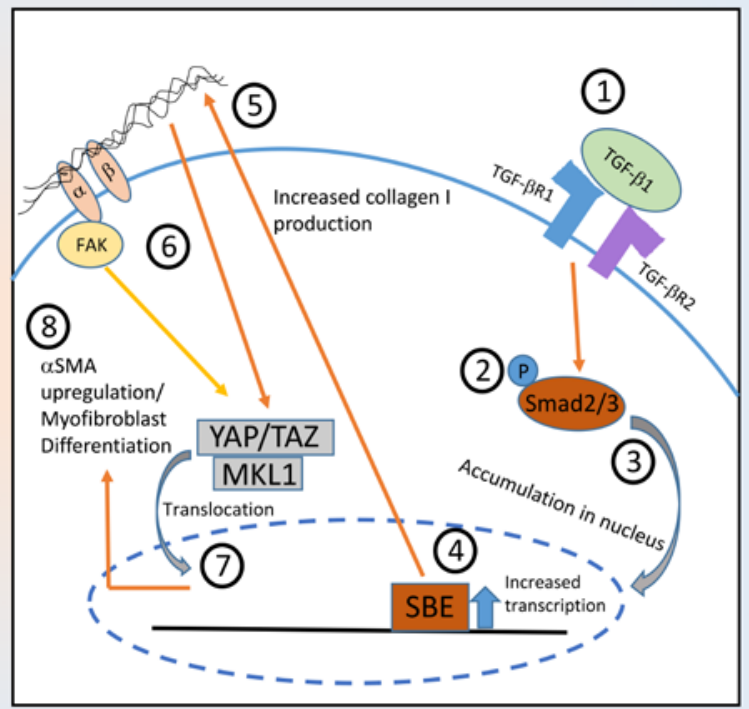

Figure 7. TGF- $\beta 1$ induced mechanotransduction pathway of $\alpha-S M A^{+}$pericyte (PC) accumulation in pulmonary fibrosis. (A) Pericyte abundant microvasculature reside in the human lung. (B) Production of TCF- $\beta 1$ by fibroblasts and resident macrophages in the interstitial space results in accumulation of $\alpha-S M A^{+} P C$ in the fibrotic foci. (C) [1] The binding of TGF- $\beta 1$ to TCF- $\beta R 1$ and TCF- $\beta R 2$ on the surface of PC initiates a Smad signaling cascade. [2] Smad2/3 gets phosphorylated and [3] translocates to the cell's nucleus. [4] The accumulation of P-Smad2/3 in the nucleus leads to increased binding to the Smad binding element (SBE), which results in increased transcription of fibrotic genes. [5] Increased production of matrix proteins, including collagen I, results in an increased ECM stiffness. [6] Focal adhesion and focal adhesion kinases (FAK) binding to the stiffened environment results in [7] increased translocation of megakaryoblastic leukemia 1 (MKL-1), yes-associated protein (YAP) and transcriptional coactivator with PDZ-binding motif (TAZ), resulting in [8] increased $\alpha$-SMA expression and myofibroblast transition.

Our results indicate that short-term exposure to TGF- $\beta 1$ is insufficient to induce $\alpha$-SMA in cultured PC. This characteristic differs markedly compared with what has been reported for resident lung fibroblasts, which are known to adopt $\alpha$-SMA expression after as little as 48 hours of TGF- $\beta 1$ exposure (33), suggesting that $\mathrm{PC}$ are a unique cell population that are functionally distinct from mature lung fibroblasts. In fact, it is after long-term exposure to TGF- $\beta 1$ that PC adopt functional properties of myofibroblasts. These data suggest several competing hypotheses: that either all PC display low-level responsiveness to TGF- $\beta 1$ that increases over time, that all PC display endogenous mechanisms to dampen TGF- $\beta 1$ activation that are lost over time, or that only a small fraction of PC are able to respond to TGF- $\beta 1$ and that they do so by producing a collagen- and fibronectin-rich ECM that drives additional accumulation of $\alpha$-SMA expressing PC via mechanotransductive responses to such ECM. Given our finding that $\alpha$-SMA ${ }^{+} \mathrm{PC}$ expand in response to biomechanical influences (Figure 7), we favor the latter hypothesis, though dedicated studies aimed at evaluating TGF- $\beta 1$ receptor expression and signaling pathways will be required to fully answer this question.

Our studies also present a potentially novel mode of action for the antifibrotic agent nintedanib. Most studies of nintedanib have considered its effect on resident lung fibroblast, and we are the first to our knowledge to propose an additional effect on PC biology. While nintedanib has been reported to inhibit tumor growth by reducing PC coverage (34), we show a connection between this triple kinase inhibitor and PC in the setting of diffuse parenchymal lung disease. Treatment with nintedanib was sufficient to reduce the expression of $\alpha$-SMA, to reduce the detection of cross-linked collagen, and to lessen the elastic modulus in long-term cultures of control lung scaffold that had been seeded with TGF- $\beta 1$ stimulated PC. These data suggest that rather than acting directly on TGF- $\beta 1$-induced myofibroblast transformation, as has been proposed for postembryonic lung fibroblasts (35), nintedanib's effects on PC might be exerted via inhibiting the amplification of fibrosis caused by the diseased ECM. This effect was partially due to MMPs, 
though other mechanisms cannot be ruled out. Further, the effect of combinatorial therapeutic approaches to trigger synergistic matrix remodeling and mechanosignaling was not evaluated in this study but may be impactful. Our data suggest that PC are capable of degenerative tissue remodeling through MMP generation observed in both vascular smooth muscle cells and fibroblasts. These PC have the capacity to behave both in a vascular role and interstitial fibroblast-like role. Further investigation of this finding may lead to better understanding of cell-matrix interactions in a variety of clinical settings beyond IPF.

\section{Methods}

Preparation of human lung. Lung pieces were frozen, sliced to $2 \mathrm{~mm}$ thickness, and rinsed with PBS containing sodium nitroprusside (SNP; Sigma-Aldrich). Slices were decellularized with 8 mM CHAPS (MilliporeSigma), $1 \mathrm{M} \mathrm{NaCl}$, and $25 \mathrm{mM}$ EDTA (Sigma-Aldrich) at $37^{\circ} \mathrm{C}$ overnight (36). Slices were treated with $90 \mathrm{U} / \mathrm{ml}$ benzonase nuclease (MilliporeSigma) for removal of cellular and nuclear content. Prepared lung tissue was then rinsed with PBS containing 10\% FBS to remove remnant DNA. After decellurization, lung slices were rinsed extensively with PBS to remove residual chemical and cellular content and sterilized with PBS containing 10\% penicillin/streptomycin (Gibco) and 2\% gentamicin (Gibco), and they were stored at $4^{\circ} \mathrm{C}$. For solubilization, lyophilized pieces were ground and digested with $1 \mathrm{mg} / \mathrm{ml}$ pepsin (MilliporeSigma) in $0.1 \mathrm{M} \mathrm{HCl}$ for 72 hours. Further, Hoescht, and $\mathrm{H} \& \mathrm{E}$ staining were conducted to determine complete removal of DNA and cellular content.

Histological and immunofluorescent evaluation. Scaffolds were fixed in 4\% paraformaldehyde, dehydrated, embedded in paraffin, and sectioned into 5- $\mu \mathrm{m}$ thick slices. Sections were stained with Masson's Trichrome (VWR International), Picrosirius red (VWR International), and $\alpha$-SMA (clone 1A4, Thermo Fisher Scientific) by sample identification-blinded facilitators at the Yale Histology Core Facility. For immunofluorescence, paraffin embedded samples were blocked with 2\% BSA and stained against NG2 (clone H-300, Santa Cruz Biotechnology Inc.), CD90 (clone K-15, Santa Cruz Biotechnology Inc.), or $\alpha$-SMA (clone 1A4, Santa Cruz Biotechnology Inc.).

Reverse transcriptase PCR on human lung fibroblasts. Fibroblasts isolated by explant outgrowth from normal donors and IPF lung explants were purchased from Asterand. Total cellular RNA was isolated, and qPCR was performed using primers against NG2 and $\beta$-actin on an Applied Biosystems 7500 Real-Time PCR System. Relative expression was determined using $2 \triangle \mathrm{CT}$ method.

$P C$ isolation and culture. Human microvascular PC were isolated from human placental tissue by microvascular segment explant outgrowth $(7,10)$, and cells were cultured in M199 (Gibco) medium supplemented with 20\% FBS (Hyclone Laboratories) and 1\% penicillin-streptomycin (Gibco). PC phenotype was verified by flow cytometry, with positive expression of NG2 (clone H-300, Santa Cruz Biotechnology Inc.), CD90 (clone K-15, Santa Cruz Biotechnology Inc.), CD146 (clone P1H12, BioLegend), and PDGFR- $\beta$ (clone 18A2, Santa Cruz Biotechnology Inc.) and no expression of SMMHC (clone N-16, Santa Cruz Biotechnology Inc.), CD31 (clone C20, Santa Cruz Biotechnology Inc.), CD34 (clone 581, MilliporeSigma), and CD45 (clone MEM-28, MilliporeSigma). As negative control, PC were incubated with isotype matched IgG.

Polyacrylamide hydrogel preparation. Amino-silanated coverslips were prepared with $0.1 \mathrm{M} \mathrm{NaOH}$, followed by 3-aminopropyltriethoxysilane (MilliporeSigma), and crosslinked with 0.05\% glutaraldehyde (Polysciences, Inc.). Chloro-silanated coverslips were prepared with dichlorodimethylsilane (MilliporeSigma) (37). Polyacrylamide hydrogels were prepared by altering the concentrations of acrylamide (Bio-Rad) and bis-acrylamide (Bio-Rad). Ammonium persulfate (MilliporeSigma) and tetramethylethylenediamine (Bio-Rad) were added, and resulting gels were hydrated in PBS. Sulfa-SANPAH (Thermo Fisher Scientific) was added in $50 \mathrm{mM}$ 4-(2-hydroxyethyl)-1-piperazineethanesulfonic acid buffer (MilliporeSigma), and gels were placed under UV (365 nm, $\left.10 \mathrm{~mW} / \mathrm{cm}^{2}\right)$ and incubated overnight with $0.5 \mathrm{mg} / \mathrm{ml}$ solubilized ECM or $0.1 \mathrm{mg} / \mathrm{ml}$ human fibronectin (MilliporeSigma) and collagen I (MilliporeSigma). Protein conjugation was validated by tagging ECM with NHS-fluorescein (5/6-carboxyfluorescein succinimidyl ester; ThermoFisher).

Tensile testing. Parenchymal regions of decellularized lung samples from control and IPF subjects, as well as polyacrylamide hydrogels of varying stiffness, were cut to $10 \mathrm{~mm}$ (length) $\times 1 \mathrm{~mm}$ (width) and analyzed using an Instron 5848 with a $10 \mathrm{~N}$ load cell. Elongation was assessed by cross-head displacement, and hydrated samples were pulled until failure at a strain rate of $1 \% / \mathrm{sec}$. The Young's modulus of tissue was determined by dividing the stress $\sigma$ by the strain $\varepsilon$, at $20 \%$ strain. Hydrogel mechanical properties were analyzed using a modified model of the Hertz equation (27). 
Evaluation of PC function. PC (passage 3-7) were seeded on control or IPF lung tissue slices, or polyacrylamide hydrogels, at $150 \times 10^{3}$ cells per sample and activated with control media (nonactivated) or $1 \mathrm{ng} / \mathrm{ml}$ TGF- $\beta 1$ (PeproTech) every 2 days for 7 days (38). TGF- $\beta 1$ concentrations were based on reports consistent with activation of fibroblasts $(39,40)$. Treatment with CCG-1423 (MilliporeSigma) was performed at day 0 for the full 7 days. For long-term differentiation studies, PC were cultured on $25 \mathrm{~mm}$ glass coverslips (VWR International) or control lung sections, and media was supplemented with $100 \mu \mathrm{M}$ ascorbic acid (Spectrum Chemicals) to induce matrix deposition with or without $1 \mathrm{ng} / \mathrm{ml}$ TGF- $\beta 1$. The media was replaced 3 times a week for 4 consecutive weeks (28 days). Treatment with nintedanib (Santa Cruz Biotechnology Inc.) was performed at day 21 for the remaining 7 days. Concentrations of CCG-1423 (1.0 nM) and nintedanib (1.0 $\mathrm{nM})$ are based on therapeutic doses in studies performed on myofibroblasts $(41,42)$.

PC were evaluated via flow cytometry using anti- $\alpha$-SMA (clone 1A4, Santa Cruz Biotechnology Inc.) and anti-vimentin (clone V630, MilliporeSigma) antibodies. Data are displayed as fold-increase, normalized to a control within each experiment. For matrix composition analysis, coverslips were stained with anti-collagen I (ab233446, Abcam), anti-laminin (ab11575, Abcam), anti-collagen IV (C1926, MilliporeSigma), or anti-fibronectin (F3648, MilliporeSigma) antibodies.

MMP detection. PC culture media was analyzed for MMPs and tissue inhibitors of metalloproteinases (TIMPs) using a Quantibody Human MMP Array 1 kit (RayBiotech). Microarray slides were scanned with a laser at $550 \mathrm{~nm}$, and the intensity of each experimental condition was normalized to standard curves.

PC culture media was analyzed for active MMP2 and - 9 by gelatin zymography. Media was diluted in 50 $\mathrm{mM}$ Tris- $\mathrm{HCl}$ (AmericanBio), $8 \%$ glycercol, 1.6\% SDS, and $0.002 \%$ bromophenol blue and resolved on a $10 \%$ acrylamide gel supplemented with $0.1 \%$ gelatin. MMPs were activated by incubating gels in $200 \mathrm{mM} \mathrm{NaCl}, 5$ $\mathrm{mM} \mathrm{CaCl} 2$ (MilliporeSigma), and $50 \mathrm{mM}$ Tris- $\mathrm{HCl}$ at $37^{\circ} \mathrm{C}$. Gels were stained with Coomassie Briliant Blue (AmericanBio Inc.), and MMP activity was assessed by quantifying reduced gelatin content in the gel.

Statistics. Data are expressed as mean \pm SEM and were analyzed for significance by 2-tailed Student $t$ test with Bonferroni post-test or one-way ANOVA with Tukey Post-Hoc test, with significance defined at $P<0.05$.

Study approval. Explanted human lung tissue was acquired from lungs obtained at autopsy from patients who had died of IPF or from nonpulmonary causes. These studies were considered "not human subjects" by the Yale IRB. PC were isolated from deidentified, discarded human tissues under protocols approved by the Yale Human Investigations Committee as described $(7,43)$.

\section{Author contributions}

PS and ALG conceived of the work; PS, ELH, ALM, and ALG wrote and edited the main manuscript; PS conducted experiments for Figures 1-6; XP, HS, and ELH conducted experiments for Figure 1; AD conducted experiments for Figure 3; and AR conducted experiments for Figure 6

\section{Acknowledgments}

We thank M. Saltzman and L. Niklason for their input. This work was supported by The Hartwell Foundation and Donna L. Dubinsky New Initiatives Award (to ALG), NIH 5T32DK101019-02 (AR), NIH R01HL109233, HL125850, and the Greenfield Foundation (to ELH).

Address correspondence to: Anjelica L. Gonzalez, 55 Prospect Street, New Haven, Connecticut 06515, USA. Phone: 203.436.2971; Email: Anjelica.gonzalez@yale.edu.

1. Wynn TA, Ramalingam TR. Mechanisms of fibrosis: therapeutic translation for fibrotic disease. Nat Med. 2012;18(7):1028-1040.

2. Booth AJ, et al. Acellular normal and fibrotic human lung matrices as a culture system for in vitro investigation. Am J Respir Crit Care Med. 2012;186(9):866-876.

3. Raghu G, Selman M. Nintedanib and pirfenidone. New antifibrotic treatments indicated for idiopathic pulmonary fibrosis offer hopes and raises questions. Am J Respir Crit Care Med. 2015;191(3):252-254.

4. Hung C, et al. Role of lung pericytes and resident fibroblasts in the pathogenesis of pulmonary fibrosis. Am J Respir Crit Care Med. 2013;188(7):820-830

5. Duffield JS, Lupher M, Thannickal VJ, Wynn TA. Host responses in tissue repair and fibrosis. Annu Rev Pathol. 2013;8:241-276.

6. Humphreys BD, et al. Fate tracing reveals the pericyte and not epithelial origin of myofibroblasts in kidney fibrosis. Am J Pathol. 2010;176(1):85-97.

7. Maier CL, Shepherd BR, Yi T, Pober JS. Explant outgrowth, propagation and characterization of human pericytes. Microcirculation. 2010;17(5):367-380. 
8. Murfee WL, Rehorn MR, Peirce SM, Skalak TC. Perivascular cells along venules upregulate NG2 expression during microvascular remodeling. Microcirculation. 2006;13(3):261-273.

9. Brown LA, Sava P, Garcia C, Gonzalez AL. Proteomic Analysis of the Pericyte Derived Extracellular Matrix. Cell Mol Bioeng. 2015;8(3):349

10. Sava P, Cook IO, Mahal RS, Gonzalez AL. Human microvascular pericyte basement membrane remodeling regulates neutrophil recruitment. Microcirculation. 2015;22(1):54-67.

11. Humphreys BD. Targeting pericyte differentiation as a strategy to modulate kidney fibrosis in diabetic nephropathy. Semin Nephrol. 2012;32(5):463-470.

12. Schrimpf C, Duffield JS. Mechanisms of fibrosis: the role of the pericyte. Curr Opin Nephrol Hypertens. 2011;20(3):297-305.

13. Parker MW, et al. Fibrotic extracellular matrix activates a profibrotic positive feedback loop. J Clin Invest. 2014;124(4):1622-1635.

14. Sun H, et al. Netrin-1 regulates fibrocyte accumulation in the decellularized fibrotic scleroderma lung microenvironment in bleomycin induced pulmonary fibrosis. Arthritis Rheumatol. 2016;68(5):1251-1261.

15. Wagner DE, et al. Comparative decellularization and recellularization of normal versus emphysematous human lungs. Biomaterials. 2014;35(10):3281-3297.

16. Engler AJ, Sen S, Sweeney HL, Discher DE. Matrix elasticity directs stem cell lineage specification. Cell. 2006;126(4):677-689.

17. Rowley JE, Johnson JR. Pericytes in chronic lung disease. Int Arch Allergy Immunol. 2014;164(3):178-188.

18. Sun $\mathrm{H}$, et al. Fibroblast engraftment in the decellularized mouse lung occurs via a $\beta 1$-integrin-dependent, FAK-dependent pathway that is mediated by ERK and opposed by AKT. Am J Physiol Lung Cell Mol Physiol. 2014;306(6):L463-L475.

19. Balestrini JL, Niklason LE. Extracellular matrix as a driver for lung regeneration. Ann Biomed Eng. 2015;43(3):568-576.

20. Balestrini JL, et al. Comparative biology of decellularized lung matrix: Implications of species mismatch in regenerative medicine. Biomaterials. 2016;102:220-230.

21. Gilbert TW, Sellaro TL, Badylak SF. Decellularization of tissues and organs. Biomaterials. 2006;27(19):3675-3683.

22. Bagley RG, et al. Pericytes from human non-small cell lung carcinomas: an attractive target for anti-angiogenic therapy. Microvasc Res. 2006;71(3):163-174.

23. Bichsel CA, Hall SR, Schmid RA, Guenat OT, Geiser T. Primary Human Lung Pericytes Support and Stabilize In Vitro Perfusable Microvessels. Tissue Eng Part A. 2015;21(15-16):2166-2176.

24. Bichsel CA, et al. Increased PD-L1 expression and IL-6 secretion characterize human lung tumor-derived perivascular-like cells that promote vascular leakage in a perfusable microvasculature model. Sci Rep. 2017;7(1):10636.

25. Maier CL, Shepherd BR, Yi T, Pober JS. Explant outgrowth, propagation and characterization of human pericytes. Microcirculation. 2010;17(5):367-380.

26. Huang X, et al. Matrix stiffness-induced myofibroblast differentiation is mediated by intrinsic mechanotransduction. Am J Respir Cell Mol Biol. 2012;47(3):340-348.

27. Lauridsen HM, Walker BJ, Gonzalez AL. Chemically- and mechanically-tunable porated polyethylene glycol gels for leukocyte integrin independent and dependent chemotaxis. Technology. 2014;133(2):1-11.

28. Hu B, Wu Z, Phan SH. Smad3 mediates transforming growth factor-beta-induced alpha-smooth muscle actin expression. Am $J$ Respir Cell Mol Biol. 2003;29(3 Pt 1):397-404.

29. Myllärniemi M, Kaarteenaho R. Pharmacological treatment of idiopathic pulmonary fibrosis - preclinical and clinical studies of pirfenidone, nintedanib, and N-acetylcysteine. Eur Clin Respir J. 2015;2.

30. Rangarajan S, et al. Novel Mechanisms for the Antifibrotic Action of Nintedanib. Am J Respir Cell Mol Biol. 2016;54(1):51-59.

31. Pu KM, Sava P, Gonzalez AL. Microvascular targets for anti-fibrotic therapeutics. Yale J Biol Med. 2013;86(4):537-554.

32. Szeto SG, et al. YAP/TAZ Are Mechanoregulators of TGF- $\beta$-Smad Signaling and Renal Fibrogenesis. J Am Soc Nephrol. 2016;27(10):3117-3128.

33. Thannickal VJ, et al. Myofibroblast differentiation by transforming growth factor-beta1 is dependent on cell adhesion and integrin signaling via focal adhesion kinase. J Biol Chem. 2003;278(14):12384-12389.

34. Kutluk Cenik B, Ostapoff KT, Gerber DE, Brekken RA. BIBF 1120 (nintedanib), a triple angiokinase inhibitor, induces hypoxia but not EMT and blocks progression of preclinical models of lung and pancreatic cancer. Mol Cancer Ther. 2013;12(6):992-1001.

35. Kimura T, et al. C-type natriuretic peptide ameliorates pulmonary fibrosis by acting on lung fibroblasts in mice. Respir Res. 2016;17:19.

36. Petersen TH, et al. Tissue-engineered lungs for in vivo implantation. Science. 2010;329(5991):538-541.

37. Tse JR, Engler AJ. Preparation of hydrogel substrates with tunable mechanical properties. Curr Protoc Cell Biol. 2010; Chapter 10:Unit 10.16 .

38. Moeller A, Ask K, Warburton D, Gauldie J, Kolb M. The bleomycin animal model: a useful tool to investigate treatment options for idiopathic pulmonary fibrosis? Int J Biochem Cell Biol. 2008;40(3):362-382.

39. Park AM, et al. Heat Shock Protein 27 Plays a Pivotal Role in Myofibroblast Differentiation and in the Development of Bleomycin-Induced Pulmonary Fibrosis. PLoS ONE. 2016;11(2):e0148998.

40. Mimura Y, Ihn H, Jinnin M, Asano Y, Yamane K, Tamaki K. Constitutive phosphorylation of focal adhesion kinase is involved in the myofibroblast differentiation of scleroderma fibroblasts. J Invest Dermatol. 2005;124(5):886-892.

41. Conte E, Gili E, Fagone E, Fruciano M, Iemmolo M, Vancheri C. Effect of pirfenidone on proliferation, TGF- $\beta$-induced myofibroblast differentiation and fibrogenic activity of primary human lung fibroblasts. Eur J Pharm Sci. 2014;58:13-19.

42. Hostettler KE, et al. Anti-fibrotic effects of nintedanib in lung fibroblasts derived from patients with idiopathic pulmonary fibrosis. Respir Res. 2014;15:157.

43. Ayres-Sander CE, Lauridsen H, Maier CL, Sava P, Pober JS, Gonzalez AL. Transendothelial migration enables subsequent transmigration of neutrophils through underlying pericytes. PLoS One. 2013;8(3):e60025. 